\section{On Compensating Long Actuator Delays in Nonlinear Control}

\author{
Miroslav Krstic
}

\begin{abstract}
We are interested in finite-escape open-loop unstable plants that are globally stabilizable in the absence of actuator delay but require controller redesign in the presence of delay. The simplest such plant is $\dot{Z}(t)=Z(t)^{2}+U(t-D)$, where $D$ is actuator delay of arbitrary length. For this system we present a control law that compensates the delay and achieves feedback linearization (of the entire ODE+delay infinite-dimensional cascade). However, even though exponential stability is achieved, the result is not global because the plant can have a finite escape with an initial condition $Z(0)>1 / D$ before the feedback control "reaches" it at $t=D$. We prove a stability result whose region of attraction is essentially $Z(0)<1 / D$ and for which we derive an asymptotic stability bound in terms of the system norm $Z(t)^{2}+\int_{t-D}^{t} U(\theta)^{2} d \theta$.
\end{abstract}

\section{INTRODUCTION}

Consider the scalar nonlinear control system

$$
\frac{d Z(t)}{d t}=Z(t)^{2}+U(t-D)
$$

where $U$ is the input, delayed by $D$ time units, and the objective is stabilization to the origin. For $D=0$ this is a trivial problem, solvable by many different feedback laws, the simplest of them being

$$
U(t)=-Z(t)^{2}-c Z(t), \quad c>0 .
$$

In this paper we consider the problem of compensating for the delay $D$ in nonlinear control design. Such a problem has been considered before for linear plants. For example, for the linear plant

$$
\dot{Z}(t)=Z(t)+U(t-D)
$$

a stabilizing control law with delay compensation is

$$
U(t)=-2 e^{D} Z(t)-2 \int_{t-D}^{t} e^{t-\theta} U(\theta) d \theta
$$

which is a $D$-compensated version of the static feedback law $U(t)=$ $-2 Z(t)$ that would achieve $\dot{Z}=-Z$ for $D=0$. This example is a special case of the general compensator design in the setting of "finite spectrum assignment" which has been pursued with great success for linear plants [1], [3], [4], [10], [11], [14], [16], and for which a complete Lyapunov function was recently provided in [9].

Control designs for nonlinear systems have been proposed by Jankovic [5], [6] and Mazenc et al. [12], [13]. These designs address systems in both strict-feedback and feedforward forms, with delays appearing at various locations in the system. Adaptive versions of predictor-based linear controllers were developed by Annaswamy

Manuscript received January 31, 2008; revised April 15, 2008. First published August 22, 2008; current version published September 10, 2008. This work was supported in part by the NSF and in part by Ford. Recommended by Associate Editor Lihua Xie.

The author is with the Department of Mechanical and Aerospace Engineering, University of California, San Diego, La Jolla, CA 92093-0411 USA (e-mail: krstic@ucsd.edu).

Digital Object Identifier 10.1109/TAC.2008.928123 et al. [2], [15]. Robustness of stability of nonlinear control systems to sufficiently small $D$, with a region of attraction "proportional" to $1 / D$, was established by Teel [19, Theorem 3].

In this paper we introduce an idea that represents, to our knowledge, the first attempt, for a nonlinear control system, at "actuator-delaycompensation" design (i.e., a full-state feedback that incorporates both the delay state and the ODE state, and recovers the nominal design when the delay is zero), with no restriction on the length of the delay. We start by stating our design first, and then "explain" it in the rest of the paper

$$
\begin{aligned}
& U(t)=-P(t)^{2}-c P(t) \\
& P(t)=\int_{t-D}^{t} P(\theta)^{2} d \theta+Z(t)+\int_{t-D}^{t} U(\theta) d \theta
\end{aligned}
$$

with an initial condition

$$
P(\theta)=\int_{-D}^{\theta} P(\sigma)^{2} d \sigma+Z(0)+\int_{-D}^{\theta} U(\sigma) d \sigma
$$

defined for $\theta \in[-D, 0]$. Note that the function $P(t)$, the ( $D$-seconds ahead) " $P$ redictor" of $Z(t)$, is given implicitly, through the nonlinear integral (6). Note also that for $D=0$ this design specializes to the "nominal" design (2). Alternative implementations of (6) as a DDE, with initial condition defined in (7), are

$$
\begin{aligned}
\dot{P}(t) & =P(t)^{2}-P(t-D)^{2}+Z(t)^{2}+U(t) \\
& =-c P(t)-P(t-D)^{2}+Z(t)^{2} .
\end{aligned}
$$

As a first iteration in explaining the control design (5) and (6), we point out that it can be proved that the feedback system (1), (5), and (6) is equivalent to the system

$$
\begin{aligned}
\dot{Z}(t) & =-c Z(t)+W(t-D) \\
W(t) & \equiv 0, \quad \text { for } t \geq 0
\end{aligned}
$$

where the function $W(\theta)$, which is possibly nonzero for $\theta \in[-D, 0]$, is defined implicitly in terms of $U(\theta)$ (understood as the "delay state," not as control) and $Z(t)$ using the nonlinear "integral-operator" relation

$$
W(\theta)=U(\theta)+P(\theta)^{2}+c P(\theta)
$$

where $-D \leq t-D \leq \theta \leq t$, and, most importantly, $P(\theta)=$ $\int_{t-D}^{\theta} P(\sigma)^{2}+Z(t)+\int_{t-D}^{\bar{\theta}} U(\sigma) d \sigma$. The inverse of (12) is also nonlinear and given explicitly as

$$
\begin{array}{r}
U(\theta)=W(\theta) \\
-\left(\int_{t-D}^{\theta} e^{-c(\theta-\sigma)} W(\sigma) d \sigma\right. \\
\left.+e^{-c(\theta-t+D)} Z(t)\right)^{2} \\
-c\left(\int_{t-D}^{\theta} e^{-c(\theta-\sigma)} W(\sigma) d \sigma\right. \\
\left.+e^{-c(\theta-t+D)} Z(t)\right) .
\end{array}
$$

The key result of the paper, besides the control design (5) and (6), is the construction of the Lyapunov function

$$
Z(t)^{2}+c \int_{t-D}^{t}(1+\theta+D-t) W(\theta)^{2} d \theta
$$


and the derivation of the stability estimates for the $L_{2}$ norm of the system state

$$
Z(t)^{2}+\int_{t-D}^{t} U(\theta)^{2} d \theta
$$

[note the difference between (14) and (15), with $W$ appearing in the former and $U$ appearing in the latter, and note that they are related through a nonlinear, infinite-dimensional transformation (13)].

\section{INFINITE-DIMENSIONAL "BACKSTEPPING TRANSFORMATION" AND ITS INVERSE}

A convenient way to study the problem (1) is using the representation where the delay state is modeled using the first order hyperbolic (transport) PDE

$$
\begin{aligned}
\dot{Z}(t) & =Z(t)^{2}+u(0, t) \\
u_{t}(x, t) & =u_{x}(x, t), \quad x \in(0, D) \\
u(D, t) & =U(t) .
\end{aligned}
$$

Note that $u(x, t)=U(t+x-D)$.

Consider the "spatially causal" (backstepping) state transformation $(u(x, t), Z(t)) \mapsto(\phi(x, t), Z(t))$, given by

$$
\begin{aligned}
& \phi(x, t)=u(x, t)+p(x, t)^{2} \\
& p(x, t)=\int_{0}^{x} p(y, t)^{2} d y+\int_{0}^{x} u(y, t) d y+Z(t)
\end{aligned}
$$

where the variable $p(x, t)$ is given implicitly in terms of $u(x, t)$ and $Z(t)$. It can be shown that the transformation (19) and (20) converts the plant (16) and (17) into

$$
\begin{aligned}
\dot{Z}(t) & =\phi(0, t) \\
\phi_{t}(x, t) & =\phi_{x}(x, t), \quad x \in(0, D) .
\end{aligned}
$$

The boundary condition for $\phi(D, t)$ is yet to be designed (it is stated below).

The $Z$-equation (16) has now been linearized but it is not asymptotically stabilized yet. To this end, we apply another transformation, $(\phi(x, t), Z(t)) \mapsto(w(x, t), Z(t))$

$$
w(x, t)=\phi(x, t)+c\left(\int_{0}^{x} \phi(y, t) d y+Z(t)\right)
$$

where $c>0$ will be used as a control gain. It can be shown that the transformation (23) converts the system (21) and (22) into

$$
\begin{aligned}
\dot{Z}(t) & =-c Z(t)+w(0, t) \\
w_{t}(x, t) & =w_{x}(x, t), \quad x \in(0, D)
\end{aligned}
$$

with a boundary condition $w(D, t)$ to be specified below.

Remark 2.1: We apologize to the reader that the transformations (19) and (20) and (23) may appear a bit like "magic." Their choice is guided by a general "backstepping" design procedure in [20] and [21], which employs Volterra series in $u$ as a function of $x$, and which simplifies to the compact form here due to the special structure that exists in the case of transport equations, but not in the case of parabolic PDEs. The "predictor" ideas can also explain the choice of the controller (5) and (6) but not the choice of the transformations (12) and (13).
Having brought the system (16) and (17) into the form (24) and (25), it remains to ensure that $w(0, t)$ goes to zero, and, in fact, that the entire infinite dimensional state $w(x, t)$ goes to zero. This is achieved with

$$
w(D, t)=0, \quad \forall t \geq 0
$$

namely, by ensuring that the "transport PDE" $(25)$ for $w(x, t)$ is fed by a zero input at $x=D$, and thus that its entire state will be "emptied out" in $D$ seconds. The condition (26) is met with the

$$
\phi(D, t)=-c\left(\int_{0}^{D} \phi(y, t) d y+Z(t)\right)
$$

which, in turn, is satisfied with the control law

$$
U(t)=u(D, t)=-p(D, t)^{2}+\phi(D, t)
$$

In summary, the control law in (28) and (27), with the help of (19) and (20), can be written as

$$
\begin{aligned}
U(t) & =-p(D, t)^{2}-c p(D, t) \\
p(D, t) & =\int_{0}^{D} p(\eta, t)^{2} d \eta+Z(t)+\int_{0}^{D} u(\eta, t) d \eta
\end{aligned}
$$

and it results in closed-loop behavior given by

$$
\begin{aligned}
\dot{Z}(t) & =-c Z(t)+w(0, t) \\
w_{t}(x, t) & =w_{x}(x, t) \\
w(D, t) & =0 .
\end{aligned}
$$

Denoting $P(t)=p(D, t)$, we get (5) and (6). From (20), for $t=0$, we get (7).

Before we discuss the transformation cascade $u(x, t) \mapsto \phi(x, t) \mapsto$ $w(x, t)$ in (19), (20), and (23), we point out that its inverse is given by

$$
\begin{aligned}
u(x, t)= & \phi(x, t)-\left(\int_{0}^{x} \phi(y, t) d y+Z(t)\right)^{2} \\
\phi(x, t)= & w(x, t) \\
& -c\left(\int_{0}^{x} e^{-c(x-y)} w(y, t) d y+e^{-c x} Z(t)\right)
\end{aligned}
$$

which can be simplified to

$$
\begin{aligned}
u(x, t)= & w(x, t) \\
& -\left(\int_{0}^{x} e^{-c(x-y)} w(y, t) d y+e^{-c x} Z(t)\right)^{2} \\
& -c\left(\int_{0}^{x} e^{-c(x-y)} w(y, t) d y+e^{-c x} Z(t)\right)
\end{aligned}
$$

and is explicit and globally well defined [if $w(x, t)$ and $Z(t)$ are bounded, $u(x, t)$ is bounded]. It is from (36) that one gets (13).

While the inverse backstepping transformation $w \mapsto u$ is well defined, the situation is not so simple for the direct transformation $u \mapsto$ $w$ given by (19), (20), and (23). The nonlinear integral (20) for the $p$-system, with $x$ as the running argument and $\int_{0}^{x} u(y, t) d y+Z(t)$ as the input, is unfortunately not solvable globally, i.e., not solvable for arbitrarily large values of $\int_{0}^{x} u(y, t) d y+Z(t)$. This failure is consistent with the fact that the system (1) is not globally stabilizable, i.e., for large initial conditions $Z(0)$ and large positive initial values of the delay state $U(t), t \in[-D, 0]$. Hence, the lack of a global result is not a failure of the method but inherent to the problem. 


\section{StABILITY}

From (31)-(33) it is clear that some form of exponential stability (less than global, but more than infinitesimally local) holds for the closed-loop system. In this section we provide an estimate of the region of attraction and an exponential stability bound. Since the gain $c$ presents us with too many agonizing choices in computing the estimates, we provide a result simply for $c=1$, in which those choices (and various forms of conservativeness associated with them) are eliminated.

Theorem 1: Consider the system (1), (5) and (6). If

$$
Z(0)+\sup _{\theta \in[-D, 0]} \int_{-D}^{\theta} U(\sigma) d \sigma<\frac{1}{D}
$$

then the following holds:

$$
\begin{aligned}
L(t) & \leq 8\left(\Lambda+2 \Lambda^{2}\right) e^{-t / 4} \\
|U(t)| & \leq 2(\sqrt{\Lambda}+2 \Lambda) e^{-t / 2}
\end{aligned}
$$

for all $t \geq 0$, where

$$
\begin{aligned}
L(t) & =Z(t)^{2}+\int_{t-D}^{t} U(\theta)^{2} d \theta \\
\Lambda & =\Lambda_{0}+\frac{1+D}{(1-D \zeta)^{4}} \Lambda_{0}^{2} \\
\Lambda_{0} & =4(1+D) L(0) \\
\zeta & =Z(0)+\sup _{\theta \in[-D, 0]} \int_{-D}^{\theta} U(\sigma) d \sigma .
\end{aligned}
$$

Proof: By applying Lemmas 1-7 from the Appendix , in the exact order given there.

\section{FAILURE OF THE UNCOMPENSATED CONTROLLER}

Theorem 2: Consider the plant (1) under the nominal controller (2). For a given $D>0$, there exist initial conditions $Z(0)$ satisfying the condition (37), i.e., not causing finite escape before $t=D$ in open loop and being within the region of attraction in closed-loop with the compensated controller (5), for which the solution of the uncompensated closed-loop system (1) and (2) escapes to infinity before $t=3 \mathrm{D} / 2$.

Proof: Take $U(\theta)=0, \forall \theta \in[-D, 0]$, and denote $Z_{0}=Z(0)$. During the time interval $[0, D]$ the solution is $Z(t)=\frac{Z_{0}}{1-Z_{0} t}$. Over the interval $[D, 2 D]$ the system is governed by $Z(t)=\int_{D}^{t} Z(\tau)^{2} d \tau+\frac{Z_{0}}{1-Z_{0} D}+\int_{D}^{t} U(\tau-D) d \tau$, where $U(t-D)=-\left(\frac{Z_{0}}{1-Z_{0}(t-D)}\right)^{2}-\frac{Z_{0}}{1-Z_{0}(t-D)}$. It can be easily shown that $\int_{D}^{t} U(\tau-D) d \tau=Z_{0}-\frac{Z_{0}}{1-Z_{0}(t-D)}+\ln \left(1-Z_{0}(t-D)\right)$. It follows then that $Z(t)^{2} \geq\left(\int_{D}^{t} Z(\tau)^{2} d \tau+\gamma\right)^{2}$, where $\gamma=\frac{Z_{0}}{1-Z_{0} D}+Z_{0}-\frac{Z_{0}}{1-Z_{0} D / 2}+\ln \left(1-Z_{0} D / 2\right)$. The system will have finite escape before $t=3 D / 2$ if $\gamma>2 / D$. Denote $\epsilon=1-Z_{0} D$. After some calculations the condition $\gamma>2 / D$ can be written as

$$
\frac{1}{2 \epsilon}>\frac{2}{1+\epsilon}+\frac{\epsilon}{2}+\frac{D}{2} \ln \frac{2}{1+\epsilon}
$$

where, as $Z_{0}$ increases towards $1 / D$, the left side goes to infinity, while the right side goes towards $2+\frac{D}{2} \ln 2$, where $D$ is fixed. Hence, the condition $\gamma>2 / D$ is satisfied and $Z(t)$ escapes to infinity before $t=3 D / 2$.
To help understand the importance of delay compensation, we consider the plant (1) under the predictor-based controller (5) and (6), with $U(\theta)=0, \forall \theta \in[-D, 0]$ and $Z_{0}<1 / D$. The closed-loop solution is

$$
Z(t)= \begin{cases}\frac{Z_{0}}{1-Z_{0} t}, & 0 \leq t<D \\ \frac{Z_{0}}{1-Z_{0} D} e^{-(t-D)}, & t \geq D\end{cases}
$$

whereas the control is

$$
U(t)=-\left(\frac{Z_{0}}{1-Z_{0} D} e^{-t}\right)^{2}-\frac{Z_{0}}{1-Z_{0} D} e^{-t} .
$$

The expressions (45) and (46) are so clear that we find it unnecessary to show them graphically. The initial condition $Z_{0} \in(0,1 / D)$ is a particularly interesting case to study. According to (45), $Z(t)$ grows aggressively until $t=D$, and then decays exponentially to zero. The control starts with a large negative value $U(0)=-\frac{Z_{0}^{2}}{\left(1-Z_{0} D\right)^{2}}-\frac{Z_{0}}{1-Z_{0} D}$, anticipating that it will need to bring $Z(t)$ down from a large value at $t=D$, and then decays exponentially. In contrast, the nominal controller, studied in Theorem 2 , starts with a much more "modest" $U(0)=-Z_{0}^{2}-Z_{0}$, not anticipating the size of $Z(D)$, and even though $U(t)$ grows over $[0, D]$, this growth is "too little, too late" to prevent finite escape.

\section{CONCLUSIONS AND DiscUSSION}

We presented a nonlinear infinite-dimensional control law for stabilization of a scalar "prototype" nonlinear system with finite escape instability in open loop, and with actuator delay of unrestricted length. The problem is not globally stabilizable (because the plant can have a blow up before any control signal has reached it), and, as shown in Theorem 2 , the "uncompensated" nonlinear controller merely delays the finite escape. In Theorem 1 we provide an estimate of the region of attraction and a transient bound for the delay-compensated nonlinear controller. The estimate of the region of attraction (37) is nonrestrictive compared to $Z_{0}<1 / D$.

One should not get sidetracked into thinking that this result requires feedback linearization. The control (5) can be replaced by the less "wasteful" feedback law $U(t)=-P(t)^{2}-P(t) \sqrt{P(t)^{2}+1}$, which does not linearize the $Z$-system, and the qualitative result of the paper would still hold. The main thing that would change is that, not only would the direct backstepping transformation (12) be implicit, but the inverse transformation (13) would also become implicit.

We want to explain our insistence on the "backstepping" analogy and terminology. We consider the term "finite spectrum assignment" to be somewhat misleading. It neglects the fact that the system $w_{t}=w_{x}$, $w(D, t) \equiv 0$, in (32) and (33), has its own spectrum, with complex poles whose real parts are at negative infinity. At any rate, stability characterization based on spectrum alone is imprecise. Instead, one should note that it can be proved that $\|w(t)\| \leq e^{b(D-t)}\left\|w_{0}\right\|$ for any $b>0$, where $\|\cdot\|$ can represent both $L_{2}[0, D]$ and $L_{\infty}[0, D]$ norms, and that the stability of the entire infinite-dimensional state of the feedback system can be characterized in the Lyapunov sense, as we did in Theorem 1 .

It is probably of interest to also elucidate the connection with the "reduction" transformation [1, Eq. (5.2) for $B_{0}=0$ ]. In the nonlinear case, we would define this transformation as (6), and one would then perform control design on (8), arriving at the controller (5) and the closed-loop system (9), with $-P(t-D)^{2}+Z(t)^{2}=0, \forall t \geq D$. The study of the closed-loop behavior would then proceed in a somewhat convoluted way, studying the behavior of $P(t)$ for $t \in[0, D]$ from (6) and for $t \geq D$ from the ODE $\dot{P}=-c P$, then interring the properties of $U(t)$ from (5), and finally deducing the properties of $Z(t)$ from (6), missing the benefits of the complete Lyapunov function (14). 
For a reader who may be new to the subject of predictor-based controllers, we point out that there exist two basic configurations, the original one by Smith [18] and the so called "modified Smith predictor" which is essentially the method of "finite spectrum assignment" [11]. The original Smith predictor structure compensates only for the predicted effect of the control input $U(t), D$ seconds in the future, without accounting for the future evolution of the system state/output $Z(t)$, and would be given in this nonlinear problem as $U(t)=-(Z(t)+\Upsilon(t))^{2}-c(Z(t)+\Upsilon(t))$, where $\Upsilon(t)=\int_{t-D}^{t} \Upsilon(\theta)^{2} d \theta+\int_{t-D}^{t} U(\theta) d \theta$, with initial condition $\Upsilon(\theta)=\int_{-D}^{\theta} \Upsilon(\sigma)^{2} d \sigma+\int_{-D}^{\theta} U(\sigma) d \sigma, \theta \in[-D, 0]$. The "modified Smith predictor" applied to our nonlinear problem is (5) and (6). The original Smith predictor is effective only for open-loop stable plants, whereas the modified Smith predictor also works for unstable plants. Observe that, when $U(\theta)=0, \theta \in[-D, 0]$, the original Smith predictor feedback produces the same input signal for $t \in[0, D]$ as the uncompensated feedback, $U(t)=-Z(t)^{2}-c Z(t)$, therefore, based on Theorem 2 , it can lead to finite escape over the interval $[D, 2 D]$.

The result of this paper seems generalizable to general nonlinear systems with actuator delay. Consider the system $\dot{Z}(t)=f(Z(t), U(t-$ $D)$ ), where $Z$ is now a vector, and suppose that a function $\kappa(Z)$ is known such that $\dot{Z}=f(Z, \kappa(Z))$ is globally asymptotically stable at the origin. Then the delay-compensating controller would be chosen as

$$
\begin{aligned}
& U(t)=\kappa(P(t)) \\
& P(t)=\int_{t-D}^{t} f(P(\theta), U(\theta)) d \theta+Z(t)
\end{aligned}
$$

with $P(\theta)=\int_{-D}^{\theta} f(P(\sigma), U(\sigma)) d \sigma+Z(0), \theta \in[-D, 0]$. The backstepping transformation and its inverse would be defined as

$$
\begin{aligned}
W(t) & =U(t)-\kappa(P(t)) \\
U(t) & =W(t)+\kappa(\Pi(t))
\end{aligned}
$$

where

$$
\Pi(t)=\int_{t-D}^{t} f(\Pi(\theta), \kappa(\Pi(\theta))+W(\theta)) d \theta+Z(t)
$$

with initial condition $\Pi(\theta)=\int_{-D}^{\theta} f(\Pi(\sigma), \kappa(\Pi(\sigma))+W(\sigma)) d \sigma+$ $Z(0), \theta \in[-D, 0]$. Note that $P=\Pi$, however they play different roles because they are driven by different inputs $(U$ versus $W$ ). The mapping (49) represents the direct backstepping transformation $U \mapsto$ $W$, whereas (50) represents the inverse backstepping transformation $W \mapsto U$. Both transformations are nonlinear and infinite dimensional. Note that the inverse transformation $W \mapsto U$ will typically be globally well defined because (51) only requires a minor extra assumption that the feedback $\kappa(\cdot)$ be ISS stabilizing with respect to $W$, in addition to being GAS stabilizing (the ISS assumption is readily satisfied with backstepping controllers for strict-feedback systems, as well as for forwarding controllers [7], [17]). The direct backstepping transformation $U \mapsto W$ will typically not be globally well defined, however, in the case of feedforward systems, which are "forward complete," it is expected to be globally well defined, and hence the delay compensation design for feedforward systems is expected to be globally asymptotically stabilizing, which is consistent with the result of [12] achieved with memoryless feedback.

\section{APPENDIX}

In what follows, we denote $Z_{0}=Z(0), w_{0}(x)=w(x, 0)$, etc. The following notation is used: $\|w(t)\|^{2}=\int_{0}^{D} w(x, t)^{2} d x$. Occasionally we will be suppressing the dependence on $t$ to save space.
Lemma 1: The following holds for (31)-(33):

$$
Z(t)^{2}+\|w(t)\|^{2} \leq 2\left(Z_{0}^{2}+\left\|w_{0}\right\|^{2}\right) e^{-t / 2} .
$$

Proof: Consider the Lyapunov functional $\Omega(t)=$ $\frac{1}{2}\left(Z(t)^{2}+\int_{0}^{D}(1+x) w(x, t)^{2} d x\right)$. Its derivative is

$$
\begin{aligned}
\dot{\Omega}= & -Z^{2}+Z w(0, t) \\
& +\int_{0}^{D}(1+x) w(x, t) w_{x}(x, t) d x \\
= & -Z^{2}+Z w(0, t) \\
& +\frac{1}{2} \int_{0}^{D}(1+x) d\left(w(x, t)^{2}\right) \\
= & -Z^{2}+Z w(0, t) \\
& -\frac{1}{2} w(0, t)^{2}-\frac{1}{2} \int_{0}^{D} w(x, t)^{2} d x \\
\leq & -\frac{1}{2} Z^{2}-\frac{1}{2} \int_{0}^{D} w(x, t)^{2} d x \\
\leq & -\frac{1}{2} Z^{2}-\frac{1}{4} \int_{0}^{D}(1+x) w(x, t)^{2} d x \\
\leq & -\frac{1}{2} \Omega
\end{aligned}
$$

so, $\Omega(t) \leq \Omega(0) e^{-t / 2}$. Noting that $Z(t)^{2}+\|w(t)\|^{2} \leq 2 \Omega(t)$ and $\Omega(0) \leq Z_{0}^{2}+\left\|w_{0}\right\|^{2}$, the lemma is proved.

Lemma 2: The following holds for (36):

$$
Z^{2}+\|u\|^{2} \leq 4\left[Z^{2}+\|w\|^{2}+\left(Z^{2}+\|w\|^{2}\right)^{2}\right] .
$$

Proof: We start by writing (36) as

$$
u(x)=w(x)-\left(\psi(x)+e^{-x} Z\right)-\left(\psi(x)+e^{-x} Z\right)^{2}
$$

where $\psi(x)=e^{-x} \star w(x)$ and $\star$ denotes the convolution operator. By squaring up (55), applying Young's inequality, and integrating in $x$, we get

$$
\begin{aligned}
\|u\|^{2} \leq & 2\|w\|^{2}+2\|\psi\|^{2}+4\left\|\psi^{2}\right\|^{2} \\
& +2\left(\int_{0}^{D} e^{-2 x} d x\right) Z^{2} \\
& +4\left(\int_{0}^{D} e^{-4 x} d x\right) Z^{4} \\
\leq & 2\|w\|^{2}+2\|\psi\|^{2}+4\left\|\psi^{2}\right\|^{2} \\
& +Z^{2}+Z^{4}
\end{aligned}
$$

From [8, Theorem B.2(ii)] it follows that $\|\psi\|^{2} \leq\|w\|^{2}$. It remains to consider the term $\left\|\psi^{2}\right\|^{2}$. We have that $\left\|\psi^{2}\right\|^{2} \leq$ $\|\psi\|^{2} \sup _{x \in[0, D]} \psi(x)^{2}$. Noting that $\psi^{\prime}=-\psi+w$, and that this implies $\left(\psi^{2}\right)^{\prime} \leq-\psi^{2}+w^{2}$, it follows from [8, Lemma B.6] that $\sup _{x \in[0, D]} \psi(x)^{2} \leq\|w\|^{2}$. Hence, $\left\|\psi^{2}\right\|^{2} \leq\|\psi\|^{2}\|w\|^{2} \leq\|w\|^{4}$ and we get

$$
\|u\|^{2} \leq 4\|w\|^{2}+4\|w\|^{4}+Z^{2}+Z^{4}
$$

The (rather conservative) bound (54) follows.

Lemma 3: The following holds for (23):

$$
\left\|w_{0}\right\|^{2} \leq 2(1+D)\left\|\phi_{0}\right\|^{2}+2 D Z_{0}^{2} .
$$

Proof: Immediate, by noting that $\int_{0}^{D}\left(\int_{0}^{x} \phi_{0}(y) d y\right)^{2} d x \leq$ $D\left\|\phi_{0}\right\|^{2}$, which follows from the Cauchy-Schwartz inequality. 
Lemma 4: Denote

$$
\zeta=Z_{0}+\sup _{x \in[0, D]} \int_{0}^{x} u_{0}(y) d y
$$

and consider the transformation (20). Then

$$
p_{0}(x)^{2} \leq \frac{\zeta^{2}}{(1-\zeta x)^{2}} .
$$

Proof: We start by noting that

$$
p_{0}(x)^{2} \leq\left(\int_{0}^{x} p_{0}(y)^{2} d y+\zeta\right)^{2}
$$

which is true because $v_{0}(x)$ is defined as a nonnegative valued function. We introduce the change of variable $r(x)=\int_{0}^{x} p_{0}(y)^{2} d y, r^{\prime}(x)=$ $p_{0}(x)^{2}$, which gives a nonlinear differential inequality $r^{\prime} \leq(r+\zeta)^{2}$, with an initial condition $r(0)=0$. By the comparison principle it follows that $r(x) \leq \rho(x)$, where $\rho(x)$ is the solution of the nonlinear differential equation $\rho^{\prime}=(\rho+\zeta)^{2}, \rho(0)=0$. Since $\rho(x)=\frac{\zeta^{2} x}{1-\zeta x}$, it follows that $\int_{0}^{x} p_{0}(y)^{2} d y \leq \frac{\zeta^{2} x}{1-\zeta x}$. By using the inequality (61), the result of the lemma follows.

Lemma 5: Consider the transformation $\left(u_{0}(x), Z_{0}\right) \mapsto \phi_{0}(x)$ defined by the expressions (19) and (20). It satisfies the following bound:

$$
\left\|\phi_{0}\right\|^{2} \leq 2\left[\left\|u_{0}\right\|^{2}+4 \frac{(1+D)^{2}}{(1-\zeta D)^{2}}\left(Z_{0}^{2}+\left\|u_{0}\right\|^{2}\right)^{2}\right]
$$

Proof: We start by observing from (19) that $\left\|\phi_{0}\right\|^{2} \leq 2\left\|u_{0}\right\|^{2}+$ $2\left\|p_{0}^{2}\right\|^{2}$. Using the estimate in Lemma 4 , we get $\left\|p_{0}^{2}\right\|^{2} \leq D \frac{\zeta^{4}}{(1-\zeta D)^{4}}$, which yields $\left\|\phi_{0}\right\|^{2} \leq 2\left(\left\|u_{0}\right\|^{2}+D \frac{\zeta^{4}}{(1-\zeta D)^{4}}\right)$. From (59) it follows that $\zeta^{4} \leq 4\left(Z_{0}^{2}+D\left\|u_{0}\right\|^{2}\right)^{2} \leq 4(1+D)^{2}\left(Z_{0}^{2}+\left\|u_{0}\right\|^{2}\right)^{2}$. Combining the last two inequalities, the lemma is proved.

Lemma 6: Denote $\Lambda_{0}=4(1+D)\left(Z_{0}^{2}+\left\|u_{0}\right\|^{2}\right)$. The following holds:

$$
Z_{0}^{2}+\left\|w_{0}\right\|^{2} \leq \Lambda_{0}+\frac{1+D}{(1-D \zeta)^{4}} \Lambda_{0}^{2}
$$

Proof: Immediate, by substituting the inequality of Lemma 5 into the inequality of Lemma 3 , and by applying the inequality $2 D Z_{0}^{2} \leq$ $4(1+D)^{2} Z_{0}^{2}$ (which is extremely conservative but we use it for simplicity of expression in the result of the main theorem).

Lemma 7: The following holds:

$$
|U(t)| \leq(2 \sqrt{\Lambda}+4 \Lambda) e^{-t / 2}
$$

Proof: With $w(D, t) \equiv 0$, from (36), we get that

$$
u(D)=-\left(\psi(D)+e^{-D} Z\right)-\left(\psi(D)+e^{-D} Z\right)^{2}
$$

where $\psi(x)=e^{-x} \star w(x)$, and hence, $|u(D)| \leq|\psi(D)|+$ $2|\psi(D)|^{2}+e^{-D}|Z|+e^{-2 D} Z^{2}$. As we noted in the proof of Lemma 2, $|\psi(D)| \leq \sup _{x \in[0, D]}|\psi(x)| \leq\|w\|$. Thus, $|u(D)| \leq\|w\|+2\|w\|^{2}+e^{-D}|Z|+e^{-2 D} Z^{2}$, which implies $|u(D)| \leq \sqrt{2} \sqrt{Z^{2}+\|w\|^{2}}+2\left(Z^{2}+\|w\|^{2}\right)$. From Lemma 1, we get $|U(t)|=|u(D, t)| \leq 2 \sqrt{Z_{0}^{2}+\left\|w_{0}\right\|^{2}} e^{-t}+4\left(Z_{0}^{2}+\left\|w_{0}\right\|^{2}\right) e^{-t / 2}$. With Lemma 6, we complete the proof.

\section{ACKNOWLEDGMENT}

The author would like to thank Drs. A. Smyshlyaev and R. Vazquez for helpful discussions.

\section{REFERENCES}

[1] Z. Artstein, "Linear systems with delayed controls: A reduction," IEEE Trans. Autom. Control, vol. 27, pp. 869879-869879, 1982.

[2] S. Evesque, A. M. Annaswamy, S. Niculescu, and A. P. Dowling, "Adaptive control of a class of time-delay systems," ASME Trans. Dynam., Syst., Meas., Control, vol. 125, pp. 186-193, 2003.

[3] Y. A. Fiagbedzi and A. E. Pearson, "Feedback stabilization of linear autonomous time lag systems," IEEE Trans. Autom. Control, vol. 31, pp. 847-855, 1986.

[4] K. Gu and S.-I. Niculescu, "Survey on recent results in the stability and control of time-delay systems," Trans. ASME, vol. 125, pp. 158-165, 2003.

[5] M. Jankovic, "Control Lyapunov-Razumikhin functions and robust stabilization of time delay systems," IEEE Trans. Autom. Control, vol. 46, pp. 1048-1060, 2001.

[6] M. Jankovic, "Control of cascade systems with time delay-The integral cross-term approach," in Proc. IEEE Conf. Decision and Control, 2006.

[7] M. Krstic, "Feedback linearizability and explicit integrator forwarding controllers for classes of feedforward systems," IEEE Trans. Autom. Control, vol. 49, pp. 1668-1682, 2004.

[8] M. Krstic, I. Kanellakopoulos, and P. V. Kokotovic, Nonlinear and Adaptive Control Design. New York: Wiley, 1995.

[9] M. Krstic and A. Smyshlyaev, "Backstepping boundary control for first order hyperbolic PDEs and application to systems with actuator and sensor delays," in Proc. Amer. Control Conf., 2007.

[10] W. H. Kwon and A. E. Pearson, "Feedback stabilization of linear systems with delayed control," IEEE Trans. Autom. Control, vol. 25, pp. 266-269, 1980.

[11] A. Z. Manitius and A. W. Olbrot, "Finite spectrum assignment for systems with delays," IEEE Trans. Autom. Control, vol. 24, pp. 541-553, 1979.

[12] F. Mazenc, S. Mondie, and R. Francisco, "Global asymptotic stabilization of feedforward systems with delay at the input," IEEE Trans. Autom. Control, vol. 49, pp. 844-850, 2004.

[13] F. Mazenc and P.-A. Bliman, "Backstepping design for time-delay nonlinear systems," IEEE Trans. Autom. Control, vol. 51, pp. 149-154, 2004.

[14] S. Mondie and W. Michiels, "Finite spectrum assignment of unstable time-delay systems with a safe implementation," IEEE Trans. Autom. Control, vol. 48, pp. 2207-2212, 2003.

[15] S.-I. Niculescu and A. M. Annaswamy, "An adaptive Smith-controller for time-delay systems with relative degree $n * \geq 2$," Syst. Control Lett., vol. 49, pp. 347-358, 2003.

[16] A. W. Olbrot, "Stabilizability, detectability, and spectrum assignment for linear autonomous systems with general time delays," IEEE Trans. Autom. Control, vol. 23, pp. 887-890, 1978.

[17] R. Sepulchre, M. Jankovic, and P. V. Kokotovic, "Integrator forwarding: A new recursive nonlinear robust design," Automatica, vol. 33, pp. 979-984, 1997.

[18] O. J. M. Smith, "A controller to overcome dead time," ISA, vol. 6, pp. 28-33, 1959.

[19] A. R. Teel, "Connections between Razumikhin-type theorems and the ISS nonlinear small gain theorem," IEEE Trans. Autom. Control, vol. 43, pp. 960-964, 1998.

[20] R. Vazquez and M. Krstic, "Control of 1-D parabolic PDEs with Volterra nonlinearities-Part I: Design," Automatica, to be published.

[21] R. Vazquez and M. Krstic, "Control of 1-D parabolic PDEs with Volterra nonlinearities-Part II: Analysis," Automatica, to be published. 